\title{
Direct detection of membrane channels from gels using water-in-oil droplet bilayers.
}

\author{
Andrew J. Heron, James R. Thompson, Amy E. Mason, Mark I. Wallace* \\ Department of Chemistry, University of Oxford, Chemistry Research Laboratory, \\ Mansfield Road, OX1 3TA, England, UK. \\ mark.wallace@chemistry.oxford.ac.uk
}

Supplementary Movie. The supplementary real-time movie shows DHBs on a $2 \%$ agarose surface, and demonstrates the various ways that the bilayers can be manipulated. Bilayer formation: this shows the zipping event of bilayer formation of a free droplet (without electrode) on the agarose surface. The bilayer forms spontaneously approximately 30 seconds after droplet addition and surface contact. Bilayer translation: this shows the droplet bilayer being translated across the surface of the agarose by moving the inserted electrode. Bilayer size control: this shows how the size of the bilayer can be adjusted by moving the inserted electrode in the vertical direction. Bilayer reformation: this shows how the bilayer can be removed and reformed, by pulling it from the surface with the inserted electrode then returning it again. This is used in the scanning experiments to remove inserted proteins and allow fresh proteins to insert when reformed. 
Supplementary Figure 1. Successive DHB scans across a hydrogel containing $\alpha-$ hemolysin. The figure shows an electrical current/time trace of successive DHB bilayer re-formation events; where the DHB is removed from the surface, moved and returned to the surface to reform the bilayer. For each bilayer formation event many $\alpha$-hemolysin channels spontaneously insert (see enlargement), producing a large peak from many stepwise increases in current. Each time the bilayer is removed the proteins are permanently lost.

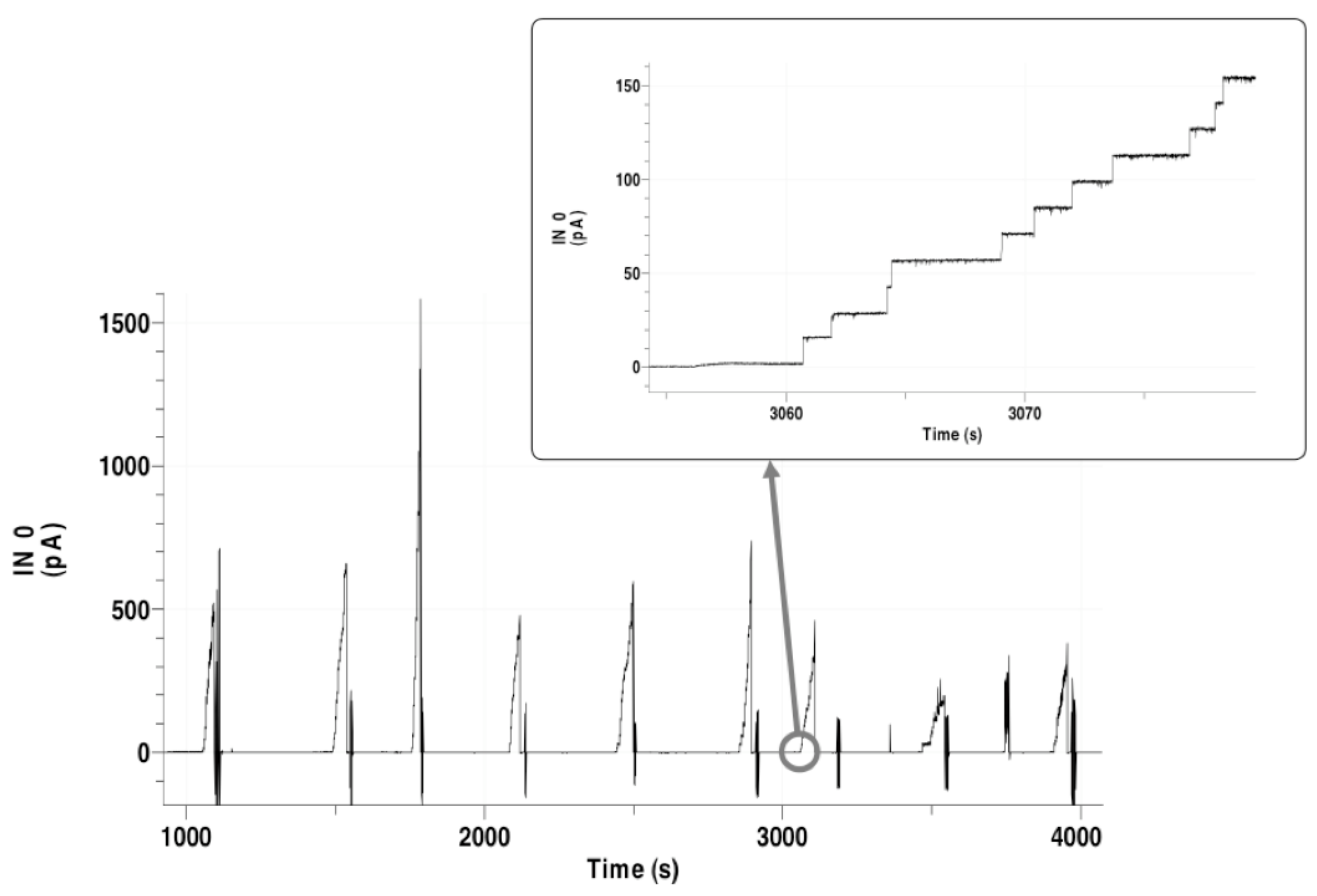


Supplementary Figure 2. Current/Voltage characteristics from single channel recording of Kcv from both droplet on hydrated support bilayers (open circles) and conventional unsupported lipid bilayers (filled circles). Unsupported MontalMueller ${ }^{14}$ lipid bilayers were formed by raising two DPhPC monolayers formed at an air-water interface over a vertical 80 micron diameter aperture in a Teflon membrane.

Data was recorded at $500 \mathrm{mM} \mathrm{KCl}$ and $\mathrm{pH} 7$.

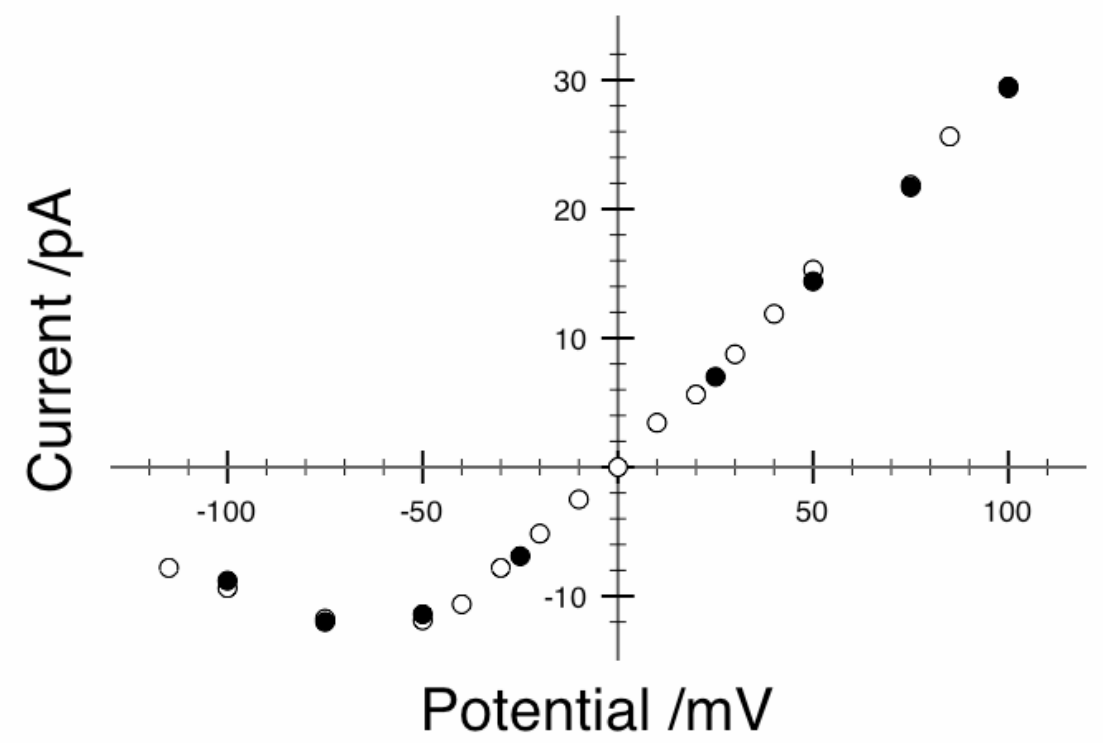


Supplementary Figure 3. SCR of Gramicidin C from Bacillus brevis. The figure shows an example of Gramicidin C (Sigma-Aldrich) channels in a DHB (-100 mV, 10 $\mathrm{mM} \mathrm{Na} \mathrm{PO}_{4}$ buffer, $\left.\mathrm{pH} 7.0,1 \mathrm{M} \mathrm{KCl}\right)$ formed on a $2 \%$ agarose gel $\left(10 \mathrm{mM} \mathrm{Na}_{\mathrm{i}} \mathrm{PO}_{4}\right.$ buffer, $\mathrm{pH} 7.0,1 \mathrm{M} \mathrm{KCl}$ ). The channels were introduced to the bilayer from the droplet side by means of vesicle fusion from gramicidin containing DOPC vesicles $(0.1 \mathrm{mg} / \mathrm{ml}$ DOPC, $5 \mu \mathrm{g} / \mathrm{ml}$ gramicidin $)$ contained within the DHB.

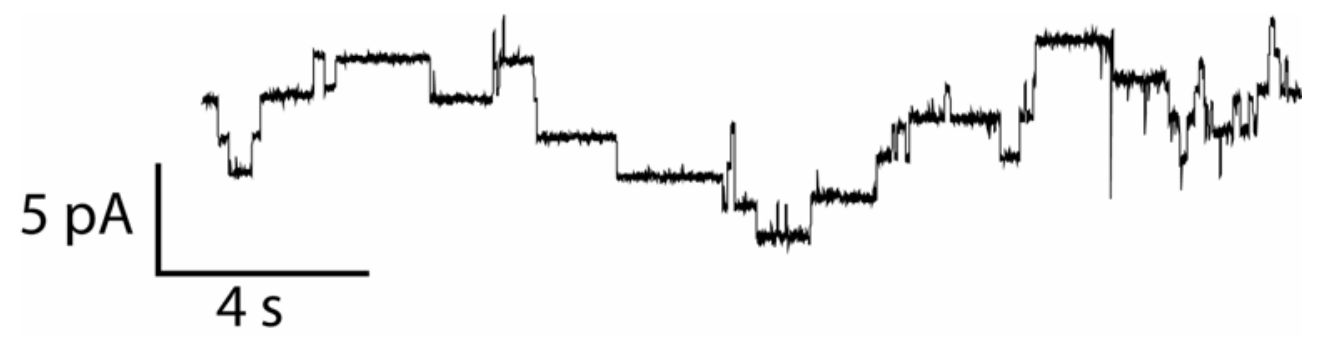


Supplementary Figure 4. SCR measurements of SDS-PAGE gels containing porin and porin free strains of E. coli. $400 \mathrm{~mL}$ cultures of JM109(DE3) and porin-free PC2889 [BL21(DE3) $\Delta$ lamB ompR] E. coli were grown in LB medium for 16 hours. The cells were harvested in a centrifuge at $2500 \mathrm{~g}$ for 20 minutes. The pellets were resuspended in $6 \mathrm{~mL} 50 \mathrm{mM}$ Tris- $\mathrm{HCl}$ buffer at $\mathrm{pH}$ 7.3. The cells were then lysed by sonication on ice. The unbroken cells were re-pelleted in a centrifuge at $2500 \mathrm{~g}$ for 10 minutes and discarded. $50 \mu \mathrm{g}$ of DNase 1 (Sigma-Aldrich) was added to the supernatant and incubated on ice for 30 minutes. The supernatant was then diluted to $60 \mathrm{~mL}$ with $100 \mathrm{mM}$ sodium carbonate at $\mathrm{pH} 11$ and stirred on ice for 1 hour. This solution was then centrifuged at an average of $\sim 120000 \mathrm{~g}$ for 1 hour. The supernatant was discarded and the membrane pellet was resuspended in $10 \mathrm{~mL} 50 \mathrm{mM}$ Tris/ $\mathrm{HCl}$ buffer at $\mathrm{pH} 7.3$ for washing. This was then recentrifuged for 30 minutes at the same speed. The membrane pellet was readied for electrophoresis by resuspension in 500 $\mu \mathrm{L}$ Tris- $\mathrm{HCl}$ buffer at $\mathrm{pH} 7.3$ and $500 \mu \mathrm{L}$ electrophoresis sample buffer containing 50\% (v:v) Glycerol and 2.5\% (w:v) SDS in Tris- $\mathrm{HCl}$ buffer at $\mathrm{pH}$ 7.3. The protein samples were loaded into a 10\% Bis-Tris polyacrylamide gels (XT Criterion; Bio-Rad Laboratories Inc.) and subjected to electrophoresis (200 V, 25 minutes) in XT MOPS buffer (Bio-Rad Laboratories Inc.). The gel tank was then refilled with SDS-free buffer (50 mM MOPS, $50 \mathrm{mM}$ Bis-Tris, $\mathrm{pH}$ 7.0), and electrophoresis was continued (100 V, 2 hours) to reduce SDS. After electrophoresis and DHB gel scanning the gel was stained with Coomassie Brilliant Blue (Sigma-Aldrich). DHB gel scanning (10 $\mathrm{mM} \mathrm{Na}_{\mathrm{i}} \mathrm{PO}_{4}, \mathrm{pH} 7.0,1 \mathrm{M} \mathrm{KCl}$ ) found no channels in the porin-free PC2889 [BL21(DE3) $\Delta$ lamB ompR] lane (lane 1) and many channels in the JM109(DE3) lane (lane 2). The figure shows some example traces acquired at $-20 \mathrm{mV}$ (top two) and $100 \mathrm{mV}$ (bottom). While the channels showed varying behavior dependent on where 
they were located on the gel, they generally shared the following porin-like characteristics: preferential insertion under high voltage, insertion as multiples (usually multiples of 3), and a tendency to shut-down under high negative voltage (e.g. bottom trace).

a

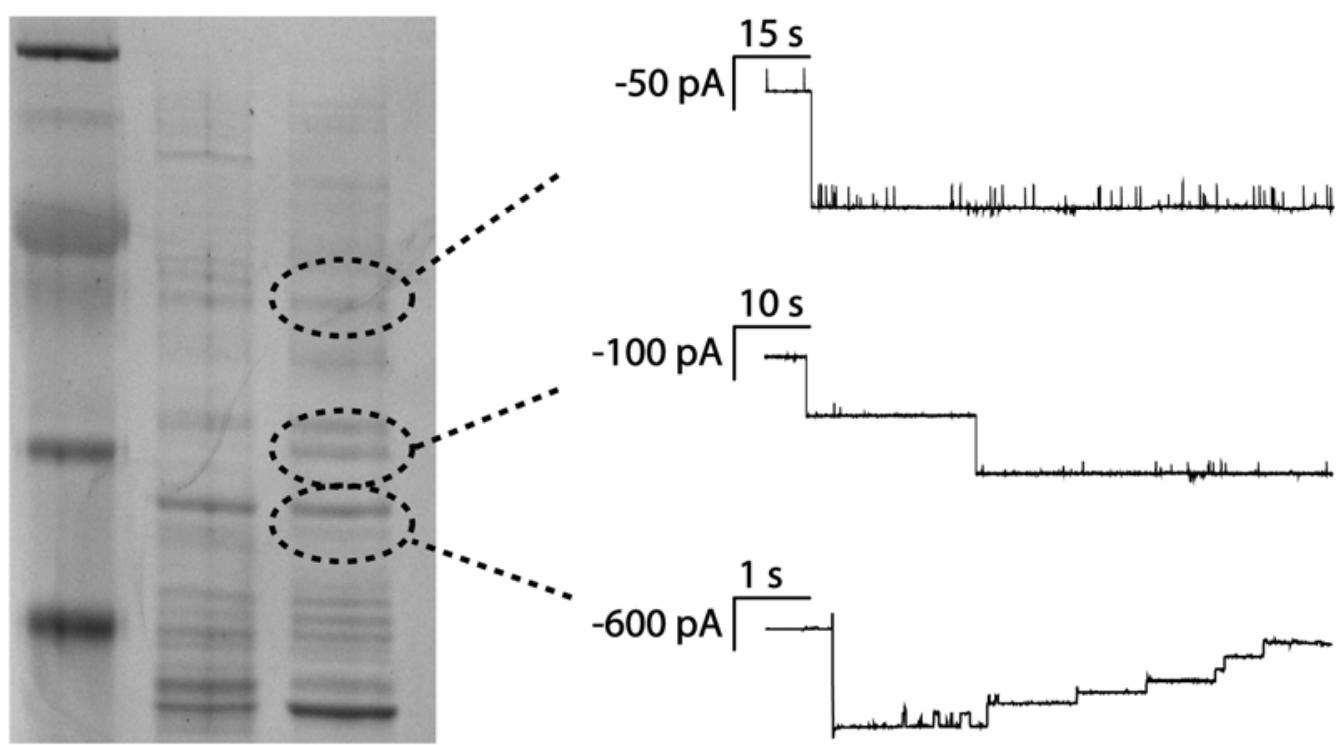

$M \quad-P \quad+P$ 